\title{
Ustilago maydis como posible bioindicador de cultivos saludables de Zea mays
}

\author{
(Ustilago maydis as possible bioindicator of healthy Zea mays crops)
}

Paúl Monar-Barragán*, Gabriela Lagla-Chimba, Stephanie López-Tipán

Universidad Central del Ecuador, Facultad de Ciencias Biológicas, Quito, Ecuador.

*Autor para correspondencia: hpmonar@uce.edu.ec

RECIBIDO: 17 de Octubre de 2019

APROBADO: 18 de Noviembre de 2019

DOI: 10.22370/bolmicol.2019.34.2.1983

LOS AUTORES DECLARAN NO TENER CONFLICTO DE INTERESES

Palabras claves: Cultivos; dispersión de esporas; maíz; polinización; Hipertrofias.

Key words: Crops, maize; spore dispersal, pollination; hypertrophy.

\section{RESUMEN}

Ustilago maydis se trata de un hongo fitopatógeno relacionado a los cultivos de maíz (Zea mays). El proceso de micosis se ve reflejada por el aparecimiento de hipertrofias de las mazorcas. Durante décadas este organismo ha sido considerado una plaga en los cultivos, sin embargo, actualmente destaca en la gastronomía Centroamericana. La baja incidencia de infección en los cultivos no transgénicos, requerimientos ambientales específicos y co-adaptación entre la especie fúngica y vegetal son características que le otorgan la capacidad bioindicadora. El presente estudio tuvo como objetivo demostrar el potencial uso de U. maydis como indicador de cultivos saludables de $Z$. mays. Se evaluaron 2 parcelas de $1000 \mathrm{~m}^{2}$ ubicadas en la parroquia Tambillo, Pichincha-Ecuador, identificando la presencia del hongo parásito en los frutos, además de la biodiversidad asociada. La presencia de infecciones micóticas en las parcelas lejos de ser indicativo de una plaga, es señal de un ambien- te propicio para el desarrollo de procesos ecológicos, entre los cuales destaca la dispersión de esporas que se encuentra vinculada a la polinización. Los resultados obtenidos a partir de las parcelas evaluadas confirman a esta especie fúngica como indicador de cultivos sanos de maíz.

\section{ABSTRACT}

Ustilago maydis is a phytopathogenic fungus related to corn crops (Zea mays). The process of mycosis is reflected by the appearance of hypertrophy (tumors) in the corncobs. For decades this organism has been considered a pest in crops, however, currently it stands out in Central American cuisine. The low incidence of infection in non-transgenic crops, specific environmental requirements and co-adaptation between fungal and vegetal species are characteristics that give it the bioindicator ability. The objective of the present study was to demonstrate the potential use of $U$. maydis as an indicator of $Z$. mays healthy crops. 
Two plots of $1000 \mathrm{~m} 2$ located in Tambillo, Pichincha-Ecuador were evaluated, identifying the presence of the parasitic fungus in the fruits, in addition to the associated biodiversity. The presence of fungal infections in the plots far from being indicative of a pest is a sign of a favorable environment to development of ecological processes, including spore dispersion that is linked to pollination. The results obtained from the evaluated plots confirm this fungal species as an indicator of healthy maize crops.

\section{INTRODUCCION}

Ustilago maydis Corda es un hongo conocido también como Huitlacoche o carbón común del maíz (Zea mays L.) ${ }^{1}$. Este hongo comestible parasitario pertenece al orden de los Ustilaginales ${ }^{2}$, infecta a granos de maíz, provocando la formación de tumores blanquecinos o grisáceos ${ }^{3,4}$. La infección a los cultivos ocurre a través de basidioesporas que infectan tejidos jóvenes del maíz, por medio de las hifas, posteriormente se forman los tumores que contienen en su interior teliosporas del hongo, las cuales al reventarse se liberan y permanecen latentes en el suelo para infectar a más plantas de maíz o cultivos ${ }^{5}$. La dispersión de las esporas se da a través del viento, sin embargo, el papel de los polinizadores es crucial para este fin. El potencial bioindicador de $U$. maydis en cultivos no ha sido evaluado, sin embargo, se estudia a este hongo por su alto valor nutricional y su importancia en la gastronomía de varios países ${ }^{5,6}$.

U. maydis únicamente se presenta bajo condiciones ambientales favorables en los cultivos de maíz: una temperatura de 17 a $20{ }^{\circ} \mathrm{C}$, humedad relativa del $80 \%$ y un óptimo nivel de nitrógeno ${ }^{7}$. La aparición de $U$. maydis depende de la salud de los cultivos ${ }^{8}$. Por otra parte, la baja cantidad de lluvia, escasa profundidad del suelo, baja fertilidad y uso de agroquímicos que provocan la erosión del suelo son los principales factores que limitan el de- sarrollo de un maíz sano y por ende del hongo ${ }^{9}$. Un cultivo saludable, presentará las condiciones ambientales, biológicas y fisicoquímicas adecuadas para la aparición de U. maydis en el maíz ${ }^{1,9}$.

\section{MATERIALES Y MÉTODOS}

\section{Área de estudio}

Se evaluaron dos parcelas de cultivos de maíz (Z. mays) no transgénico de $1000 \mathrm{~m}^{2}$ cada una, pertenecientes a la hacienda "Tambillo Viejo" ubicada en la parroquia Tambillo del cantón Mejía, provincia Pichincha. En cada parcela existía un aproximado de 350 individuos plantados. La Parroquia de Tambillo posee formaciones vegetales correspondientes a Bosque Montano Bajo, sin embargo, la localidad estudiada al tratarse de una zona poblada carece de remanentes nativos de vegetación, posee una altitud promedio de 2700 msnm, con temperaturas fluctuantes entre los 11.9 $-21.5{ }^{\circ} \mathrm{C}$ y presencia permanente de agua para regadíos $^{10}$. La población se dedica principalmente a actividades agropecuarias. Las parcelas se localizaban a aproximadamente a $1 \mathrm{Km}$ del río San Pedro, a $750 \mathrm{~m}$ de la vía Panamericana y separadas $200 \mathrm{~m}$ entre sí. La primera parcela se ubicó en las coordenadas: $-0.40828^{\circ}$; $-78.54934^{\circ}$ a la cual se identificó con el código ZM1 y la segunda parcela con coordenadas: $-0.40902^{\circ}$; $-78.54943^{\circ}$, correspondiente al código ZM2 (Figura 1).

\section{Levantamiento de información}

Para conocer la percepción local sobre $U$. maydis y el manejo de las parcelas de Z. mays evaluadas se utilizó la herramienta de entrevistas semiestructuradas, dirigidas a los trabajadores encargados de los cultivos.

\section{Recolección y Procesamiento de muestras}

Se colectaron todos los individuos de $U$. maydis presentes en las parcelas, se colocaron en fundas de papel periódico para su transporte y procesamiento. El proceso de secado se realizó en el 

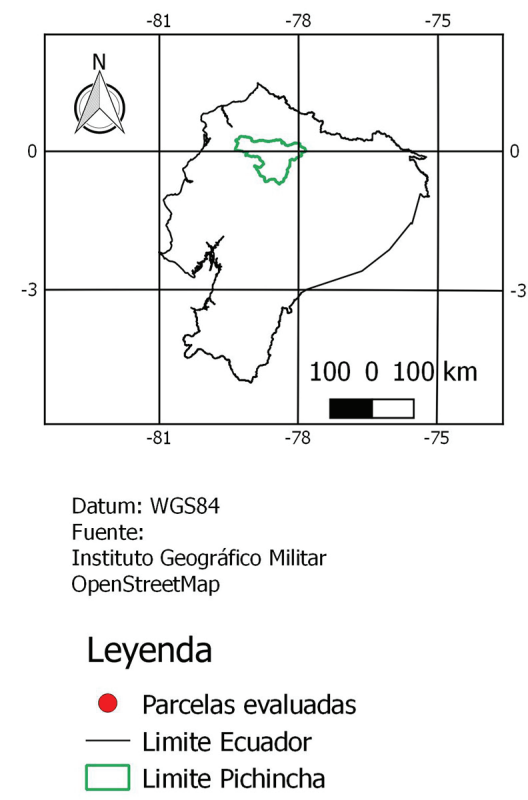

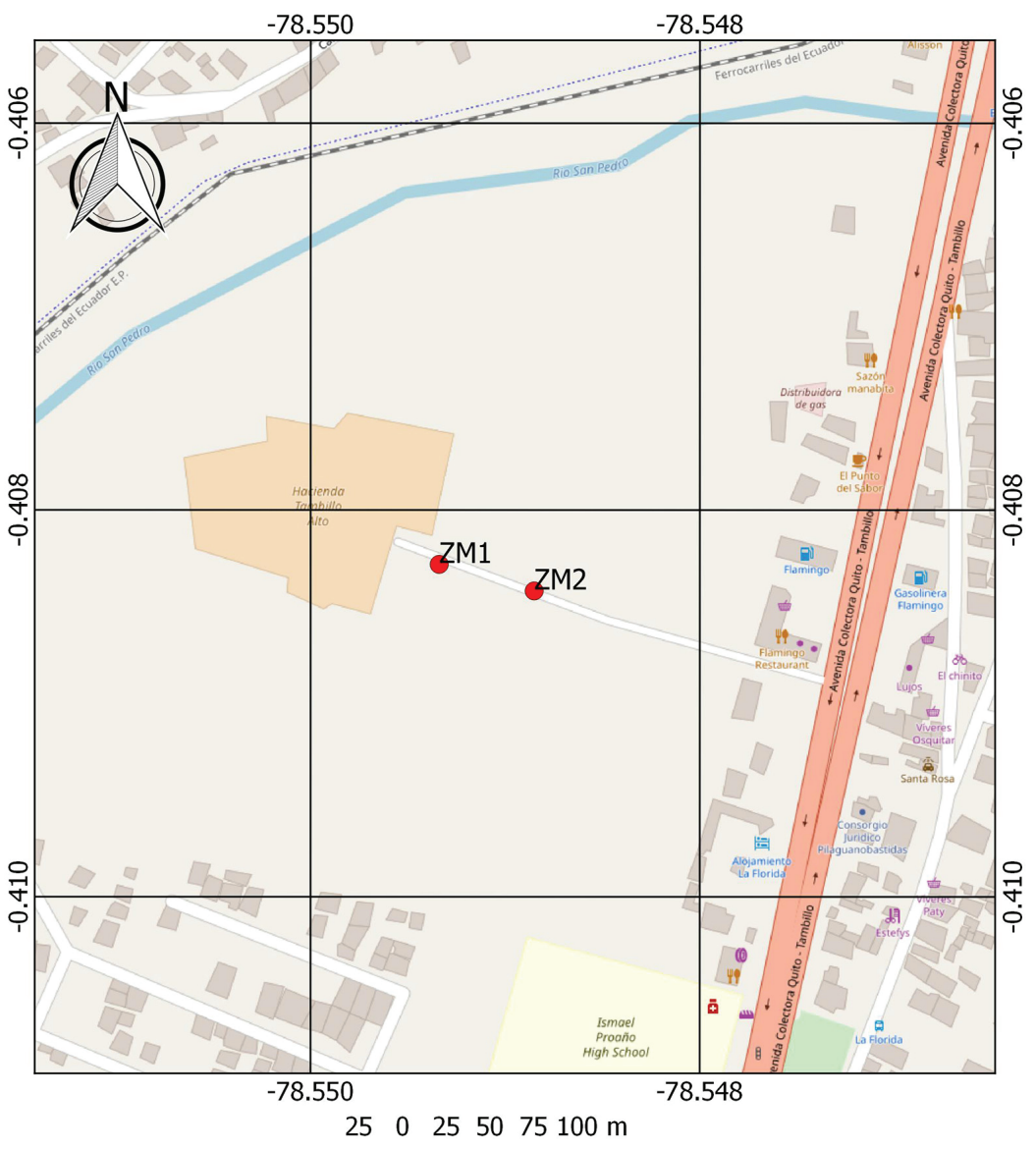

Figura 1: Ubicación del área de estudio, hacienda "Tambillo Viejo".

laboratorio de la Facultad de Ciencias Biológicas de la Universidad Central del Ecuador mediante la utilización de un equipo de secado Memmert C412.2656, durante 50 horas a $20^{\circ} \mathrm{C}$. Para la identificación se recurrió a observaciones macroscópicas de los tumores colectados, en el laboratorio de Micología Aplicada de la Universidad Central del Ecuador.

Adicional a este proceso se recolectaron especímenes de las plantas asociadas a cada una de las parcelas estudiadas. Las muestras colectadas fueron prensadas y secadas para su posterior identificación. Se recurrió a textos y guías fotográficas de la región, además de comparaciones con colecciones botánicas locales. La fauna asociada fue identificada in situ mediante observación directa e información proporcionada por los trabajadores de la hacienda.

\section{RESULTADOS}

La parcela ZM1 fue reconocida por los entrevistados como un cultivo poco saludable, se identificó el uso de herbicidas en el proceso inicial de manejo de las especies vegetales silvestres, sin embargo, han relegado a los individuos de maíz, ocupando mayor espacio del área de siembra e impidiendo el correcto desarrollo de las plantas de maíz por lo cual mantiene un tamaño de aproximadamente $50 \mathrm{~cm}$ además de carecer de mazorcas pese a encontrarse en temporada de cosecha. La parcela estaba dominada por las especies Amaranthus pubescens (Amaranthaceae), Sonchus sp. 
(Asteraceae) y varias especies del género Brassica (Brassicaceae). La información proporcionada por los trabajadores de la hacienda, indica como normal la presencia de estas especies; sin embargo, en el caso de la parcela existía un crecimiento desmedido, superior al de los individuos de $Z$. mays dejándolos inviables para su desarrollo y producción. Los polinizadores observados correspondían solamente a individuos de Apis mellifera, cuya relación era afín con las flores de las plantas silvestres descritas en lugar de las flores de Z. mays (Figura 2).

En la parcela ZM2 se colectó 3 individuos $(0.85 \%)$ de $U$. maydis, ubicados de manera heterogénea en el cultivo. La percepción de los entrevistados frente a esta especie es positiva ya que no lo consideran una plaga y por el contrario son considerados indicadores de salud en el cultivo; sin embargo, las mazorcas infectadas son descartadas. El cultivo correspondía a la variedad de maíz denominada localmente como Morochillo. Se utilizó semillas nativas que no han sufrido procesos de hibridación, provenientes de cosechas anteriores. Los individuos del cultivo alcanzaban una altura de aproximadamente $3 \mathrm{~m}$ y reconocidos en conjunto como una parcela saludable (Figura 2). Las especies silvestres asociadas en este caso corresponden a la familia Solanaceae, especialmente $\mathrm{So}$ lanum nigrescens. En relación a la fauna asociada fue posible observar a $A$. mellifera y diversas aves entre las que destacan: Pheucticus chrysogaster (Huiracchuro), Turdus fuscater (Mirlo), Zenaida auriculata (Tórtola); evidenciando consumo de las mazorcas por parte de las mismas (Figura 3).
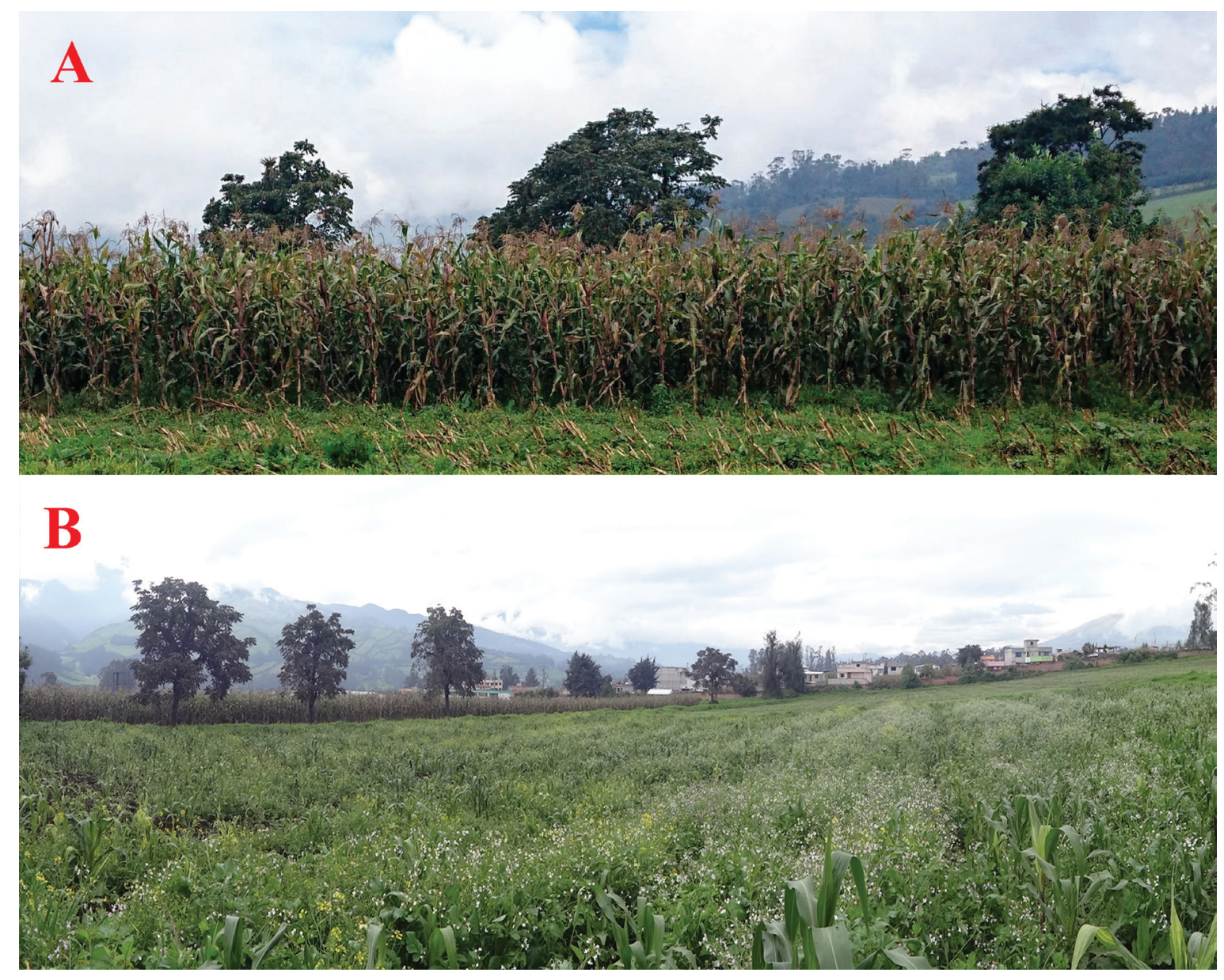

Figura 2: Cultivos de maíz con vegetación asociada. A: parcela ZM2; B: parcela ZM1. 


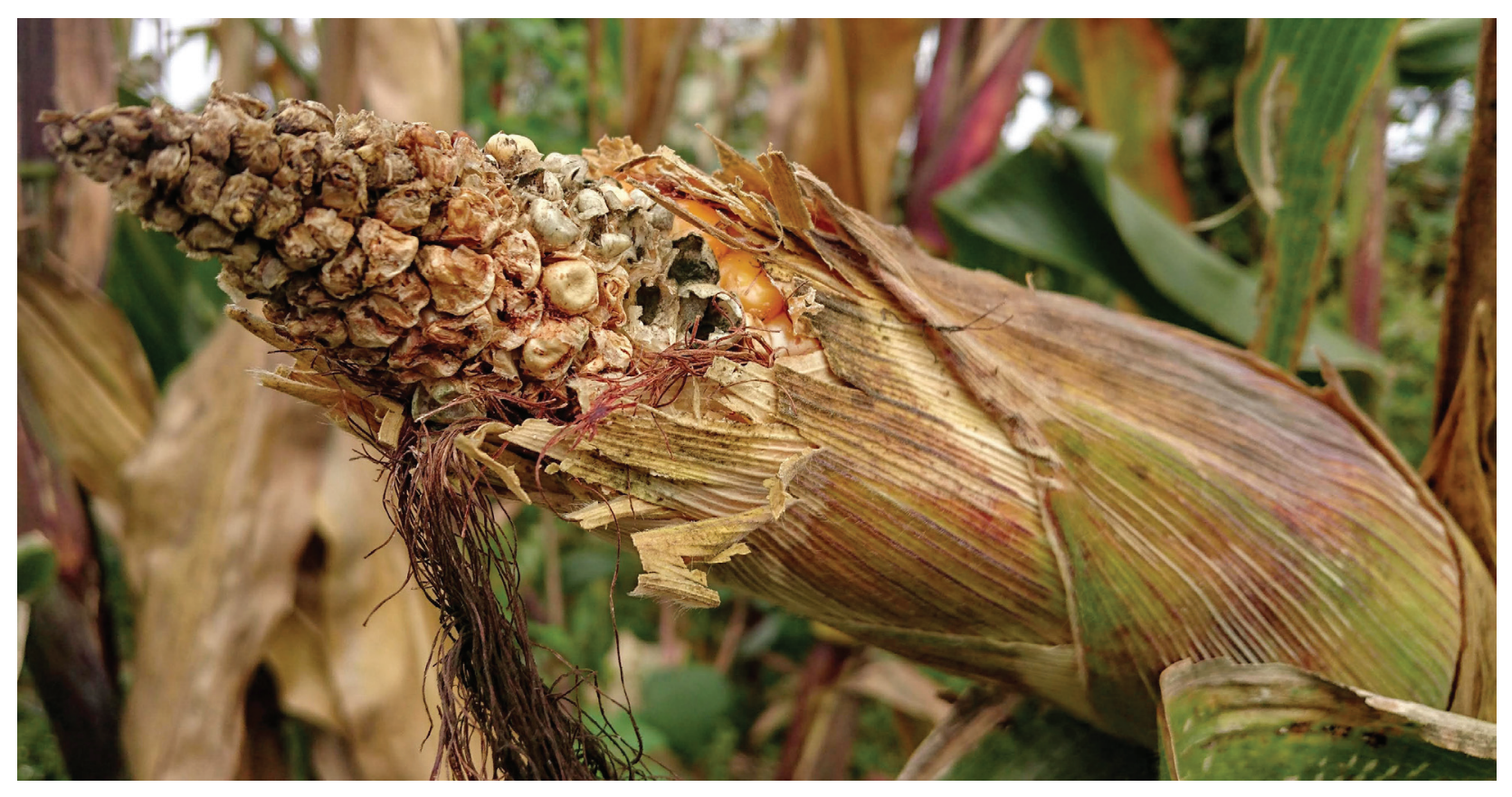

Figura 3: Mazorca de Z. mays consumida por aves silvestres.

\section{DISCUSIÓN}

Las parcelas evaluadas presentaron diferencias en cuanto a la diversidad biológica asociada, destacando la presencia de mazorcas infectadas por U. maydis. La alta especificidad a Z. mays como hospedero, indica un proceso co-adaptativo durante la domesticación del maíz, lo cual obliga al hongo a cumplir parte de su ciclo de vida en el mismo $^{5,11}$. La presencia de esporas altamente especializadas, genera dependencia a los procesos ecológicos propios de los cultivos, vinculados con la polinización que comparte escenario con el proceso de dispersión de esporas ${ }^{12,13}$. El ciclo de vida dimórfico, presenta una primera etapa (haploide) no infecciosa y la segunda etapa que requiere la presencia de plantas de maíz para la formación de micelio dicariótico ${ }^{12,14,15}$. El ingreso de las esporas puede ocurrir en cualquier órgano aéreo de las plantas de maíz pero se da mayoritariamente en las flores femeninas siendo los estigmas la vía principal de ingreso ${ }^{14,16}$, esta característica apoya la idea de un vínculo directo con la polinización especialmente por vectores bióticos.
El proceso complejo de germinación es controlado por un sistema de unión tetrapolar que solamente ocurre bajo condiciones ambientales favorables $^{2,16,17,18}$. Como organismos parásitos en su etapa diploide, $U$. maydis selecciona hospederos de un crecimiento vigoroso ${ }^{13}$ y capaces de proveer suficiente nitrógeno para la formación de hipertrofias ${ }^{15}$, tal como se pudo evidenciar en la parcela ZM2 la cual poseía individuos con un tamaño considerable. Las variedades de maíz presentan resistencia a los hongos sin embargo, en cultivos no transgénicos la tasa de infección es baja entre $1-5 \%{ }^{16}$, por lo cual no llega a considerarse una plaga, esto debido a la diversidad genética que presentan los individuos de Z. Mays ${ }^{7,19}$. En la parcela ZM2 la tasa de infección fue baja $(0.85 \%)$, esta es percibida de manera positiva por los trabajadores de la hacienda, es considerada indicadora de un cultivo saludable y con buenas condiciones ambientales. Pese a la resistencia propia de la especie hospedera el aspecto genético no es el principal en cuanto a la determinación del ciclo de vida de U. maydis; las condiciones ambientales además de la presencia de otros microorganismos patógenos determinan los 
procesos de dispersión e infección que se ven reflejado con el aparecimiento de hipertrofias $27,14,16,17,20$. La temperatura es una variable determinante en el desarrollo del hongo, $20^{\circ} \mathrm{C}$ es una condición favorable para la propagación del mismo en cultivos de maíz ${ }^{7}$. Sin embargo, la temperatura ambiental del área es inferior a este valor, lo cual puede incidir en el bajo porcentaje de infección de U. maydis.

Las aves cumplen un rol ecológico importante dentro de los procesos de polinización, siendo uno de los grupos principales vinculados a la dispersión de semillas y polen ${ }^{21,22}$, quienes indirectamente facilitan el proceso de dispersión de esporas fúngicas. La polinización por aves como la familia Trochilidae están generalmente asociadas a plantas neotropicales; pero aves perchadoras como $P$. chrysogaster (Huiracchuro) que tienen un comportamiento oportunista ${ }^{23}$, están vinculadas de manera directa con los campos de cultivo a nivel local $^{24}$. Su presencia está determinada por las características fisionómicas del hábitat ${ }^{25}$, siendo así uno de los factores importantes que contribuye a la aparición de $U$. maydis en los cultivos de maíz. $P$. chrysogaster (Huiracchuro) y Z. auriculata (Tórtola) presentan afinidad por los granos de maíz; si bien no son polinizadores principales, su contacto directo con las mazorcas y los tumores desarrolladas en las mismas, los convierten en excelentes dispersores de las esporas fúngicas.

El desarrollo de procesos ecológicos equilibrados permite que ocurran los procesos de in- fección en los individuos de maíz de las parcelas; sin embargo, las condiciones ambientales también tienen un papel clave en el desarrollo de este hongo, por lo cual es necesario evaluar estas variables de manera cuantitativa en investigaciones futuras.

\section{CONCLUSIONES}

La alta especificidad de $U$. maydis con $Z$. mays en conjunto con sus demandas ambientales, necesarias en el proceso de dispersión e infección, lo convierte en un organismo bioindicador de buena calidad de cultivos. Contrario a la percepción de plaga, la presencia de hipertrofias en las mazorcas de parcelas cultivadas, es indicadora de un ambiente con condiciones adecuadas, que además carece de otros patógenos capaces de afectar al maíz. La baja tasa de infección registrada puede deberse a la temperatura del área de estudio. Se desconoce la influencia de la altitud en el desarrollo de la especie fúngica, por lo que se recomienda tomar en cuenta esta variable.

\section{AGRADECIMIENTOS}

Agradecemos a Paúl Gamboa por su apoyo en la identificación de los individuos de $U$. $m a$ ydis y permitir el uso del laboratorio de Micología Aplicada de la Universidad Central del Ecuador, también al señor Fausto Farez-Cueva, quién facilitó el acceso y evaluación de las parcelas de maíz en la hacienda "Tambillo Viejo".

\section{REFERENCIAS}

1. Carrillo M, Tirado G, Guevara F, Héctor S, Tirado D, Miranda L. Potencial Productivo Y Caracterización Nutrimental De Dos Inóculos De Huitlacoche (Ustilago Maydis) En Variedades De Maíz. XXXIX Reunión Anual de la Asociación Mexicana de Producción Animal y Seguridad Alimentaria, A. C.; 2011 Abril 4-6; Chapingo, México.
2. Kahmann R, Steinberg G, Basse C, Feldbrügge M, Kämper J. Ustilago maydis, The causative Agent of Corn Smut Disease. En: Kronstad J, editor. Fungal Pathology. Dordrecht: Springer; 2000. p. 347-71.

3. Cabrera-Ponce J, León-Ramírez C, Verver A, Ruiz-Herrera J. Ustilago maydis. Estudios en 
maíz. En: Symposium Los microorganismos y su importancia Biotecnologica y Ecológica y la reunión regional de la zona Centro, sobre Recursos Genéticos Microbianos. Agosto 2009. Morelia, Michoacán-México.

4. Banuett F. Genetics of Ustilago maydis, A Fungal Pathogen that Induces Tumors in Maize. Annu Rev Genet. 1995; 29(1):179-208.

5. Meiners R. El cultivo de cuitlacoche (Ustilago maydis) en invernadero por los campesinos de San Miguel de Xochitecatitla, Natívitas, Tlaxcala. Universidad Nacional Autónoma de México; 2015.

6. Martínez-Flores A, Corrales-García J, Espinosa-Solares T, García-Gatica P, Villanueva-Verduzco $\mathbf{C}$. Cambios postcosecha del hongo comestible huitlacoche (Ustilago maydis (d. C.) Corda). Rev Chapingo Ser Hortic. 2008;14(3):339-46.

7. Aguayo-González DJ, Acosta-Ramos M, Pérez-Cabrera LE, Guevara-Lara F, GarcíaMunguía AM. Producción natural de huitlacoche [Ustilago maydis ( DC ) Corda] en el estado de Aguascalientes. Rev Mex Ciencias Agrícolas. 2016;7(5):1043-50.

8. Basantes E. Manejo de Cultivos Andinos del Ecuador. 1a ed. Ecuador: Andrade D, editor. Universidad de las Fuerzas Armadas ESPE; 2015. 143 p.

\section{Pimentel-González D, Rodríguez-Huezo M,} Campos-Montiel R, Trapala-Islas A, Hernández-Fuentes A. Influencia De La Variedad De Maíz En Las Características Fisicoquímicas del Huitlacoche (Ustilago maydis). Rev Mex Ing Química. 2011;10(2):171-8.

10. Gobierno Autónomo Descentralizado Municipal del Cantón Mejía. Actualización Del Plan De Desarrollo Y Ordenamiento Territorial 2015-2025 [Internet]. 2014 [citado el 16 de Julio de 2019];
Disponible en: http://www.municipiodemejia.gob. ec/documents/ordenanzas/act-pdot-2015.pdf

11. Munkacsi AB, Stoxen S, May G. Ustilago maydis populations tracked maize through domestication and cultivation in the Americas. Proc R Soc B. 2008; 275:1037-46.

12. Vollmeister E, Schipper K, Baumann S, Haag C, Pohlmann T, Stock J, et al. Fungal development of the plant pathogen Ustilago maydis. FEMS Microbiol Rev. 2012; 36(1):59-77.

13. Valverde ME, Paredes-Lapez O, GuevaraLara F, Pataky JK. Huitlacoche (Ustilago maydis) as a Food Source - Biology, Composition, and Production. Crit Rev Food Sci Nutr. 1995; 35(3):191-229.

14. Pataky JK, Snetselaar KM. Common smut of corn. Plant Heal Instr [Internet]. 2006 [citado el 13 de julio de 2019]; Disponible en: https:/www. apsnet.org/edcenter/disandpath/fungalbasidio/pdlessons/Pages/CornSmut.aspx

15. Horst RJ, Doehlemann G, Wahl R, Hofmann J, Schmiedl A, Kahmann R, et al. Ustilago maydis Infection Strongly Alters Organic Nitrogen Allocation in Maize and Stimulates Productivity of Systemic Source Leaves. Plant Physiol. 2010;152(1):293-308.

16. Ruiz J. Ustilago maydis: Ascenso de un hongo mexicano de la gastronomía local al mundo científico. Nov Sci. 2008;1(1):118-35.

17. Brefort T, Doehlemann G, Mendoza-mendoza A, Reissmann S, Djamei A, Kahmann R. Ustilago maydis as a Pathogen. Annu Rev Phytopathol. 2009;47:423-45.

18. Kahmann R, Kämper J. Ustilago maydis: how its biology relates to pathogenic development. New Phytol. 2004;164(1):31-42. 
19. El Huitlacoche [Ustilago maydis (C.D.) Corda], Alimento Prehispánico Mexicano y su Producción en Ocho Maíces Criollos de Zonas Altas. VII Congreso Internacional de Agroecología; 2018 Mayo 30, 31 y Junio 1; Córdoba, Argentina: Asociación para la Investigación en Sociología y Estudios Campesinos.

20. Pan JJ, Baumgarten A, May G. Effects of host plant environment and Ustilago maydis infection on the fungal endophyte community of maize. New Phytol. 2008;178:147-56.

21. Zaccagnini M, Thompson J, Calamari N, Goijman A, Canavelli S. Riqueza, ocupacional y roles funcionales potenciales de las aves en relación a los usos de la tierra y la productividad de los agroecosistemas: Un ejemplo en la ecoregión Pampeana en: Laterra P, editor. Valoración de servicios ecosistémicos: Conceptos, herramientas y aplicaciones para el ordenamiento territorial. Instituto Nacional de tecnologia agropecuaria: INTA; 2011. 185-219

22. Salinas L, Arana C, Pulido V. Diversidad, abundancia y conservación de aves en un ecosistema del desierto de Ica, Perú. Rev peru.biol. 2007;13(3):155-168.

23. Quesada G, Bolaños S, Cascante A. Aves perchadoras como polinizadores del árbol del dosel Bernoullia flammea (Malvaceae) en el Valle Central de Costa Rica, Brenesia. 2012;77(1): 343-350.

24. Carrión J, Aliaga M, Araujo M. El Jardín emplumado. Guía para atraer y observar aves en los jardines de Quito. 2016 p. 25.

25. Berlanga H. Conservación de las aves de América del Norte. Biodiversitas. 2001;6(38):1-8 